\title{
Prevalence of Pseudoexfoliation among Adults and its Related Ophthalmic Variables in the Community of Kebena Woreda, Gurage Zone, Ethiopia
}

SADIK Taju SHERIEF ( $\sim$ goge4000@yahoo.com )

Addis Ababa University School of Medicine https://orcid.org/0000-0003-4614-4563

Ephrem Kibru

Addis Ababa University School of Medicine

Menen Ayalew Shibeshe

Addis Ababa University School of Medicine

Research article

Keywords: pseudoexfoliation, intraocular pressure

Posted Date: November 13th, 2019

DOI: https://doi.org/10.21203/rs.2.17265/v1

License: (c) (i) This work is licensed under a Creative Commons Attribution 4.0 International License.

Read Full License 


\section{Abstract}

Clinical studies in the hospital settings using patients having ocular morbidities have shown a high prevalence of pseudoexfoliation (PXF) in Ethiopia. With this information in mind community based study was conducted to determine age and sex-specific prevalence of pseudoexfoliation syndrome (PXF) and its relationship with some ophthalmological variables. Objective To determine the prevalence and clinical features of pseudoexfoliation among adults in kebena woreda of Gurage zone, SNNPR, Ethiopia.Design Random cross-sectional samples of adult population aged 40 years or older in the community of the district.Methods $A$ total of 760 subjects aged 40 years or older underwent standardized examination, including portable slit lamp biomicroscopy before and after pupillary dilatation, and IOP measurement using Tono-Pen. PXF was diagnosed on slit lamp exam by the presence of white dandruff-like material on the pupillary margin and/or on the anterior lens capsule of one or both eyes. Results Among 760 participants, the prevalence of PXF was 12.0\% (95\% confidence interval, 9.7\%-14.3\%). The mean age was 63.9 years (SD 9.96, age range $40-90$ years). Fifty six percent were found to have bilateral PXF. The prevalence increased with increasing age, with $26.9 \%$ of those 60 or older affected. Slightly higher proportion of males $(12.4 \%)$ were found to have pseudoexfoliation in either of the eyes than females $(11.6 \%)$ which was not statistically significant $(p=0.738)$. Mean IOP in subjects with PXF was found to be $20.65+5.15 \mathrm{mmHg}$, while it was $15.0+2.3 \mathrm{mmHg}$ for those without PXF. The difference between the two populations was found to be statistically significant $(P<0.05)$.Conclusions The prevalence of PXF in eyes of people in Kebena appears greater than that reported in other places of Africa and Asia. PXF occurs at a relatively younger age in our population. Increasing age is associated with the presence of PXF, and PXF in turn is associated with high IOP.

\section{Background}

Pseudoexfoliation (PXF) is characterized by the accumulation of extracellular fibrillar material in many ocular and systemic tissues (1) and is often associated with glaucoma (2).

Pseudoexfoliation is an age-related syndrome with wide geographic variations in prevalence (3-9), even within the same population (7). The differences in prevalence have not been well explained. These variations may be either a true biological, ecological, or may even be differences related to examination techniques and diagnostic abilities.

A high prevalence of PXF in a developing country, with a large proportion of blindness due cataract and glaucoma is important for three reasons. The first is that pseudoexfoliation is associated with weak zonules and an increased risk for complications during cataract surgery (10), secondly; a clinical observation of PXF may be used as a marker to identify an individual at risk of glaucoma. Finally, an association between pseudoexfoliation and angle-closure glaucoma $(10,11)$ has been found in some ethnic groups and PXF may act as a marker to identify those with this form of glaucoma. 
Clinical studies in hospital settings in Ethiopia using patients having ocular morbidities have shown high prevalence of PXF (12-14). A recent unpublished hospital based study suggested that pseudoexfoliative glaucoma as the second most common cause of chronic glaucoma in 37\% (14). No population based prevalence studies of PXF have been published from Ethiopia. A study in South Africa revealed a prevalence of $0.08 \%$ in adults over the age of 40 (15). It is unclear whether the general population in Ethiopia has a high prevalence of PXF.

This study aimed to determine the prevalence of pseudoexfoliation in a community in southern Ethiopia among adults of 40 years and older and identify its relationship to some ophthalmic variables

\section{Methods}

This was a cross sectional Community based study that was conducted in Kebena woreda of the Gurage Zone, Ethiopia, from January 2017 to February 2017. This district is located 155 Kilo meters from Addis Ababa and consisted of 23 kebeles. The total population of the area was 70,839 for the year 2017. There were estimated 14,000 households and the mean household size was 5 people. The study population was Residents of Kebena district who were 40 years and older.

\section{Sample size Calculation}

Using a prevalence of 0.08 (taken from the population-based study in South Africa) (15), a margin of error of $2 \%$, and a confidence limit of $95 \%$ and a non response rate of $15 \%$, a sample size of 784 was established.

\section{Sampling Strategy}

\section{Sampling technique}

Multi stage sampling with probability proportionate to size was employed to select the sample. Kebeles were taken as first stage sampling units. A kebele is the smallest administrative unit of Ethiopia. Each kebele consists of at least five hundred families, or the equivalent of 3,500 to 4,000 persons. It is part of a woreda. The list of current kebeles in the woreda was obtained from the local administration and a random sample of 10 kebeles was taken from all the 23 kebeles in the Woreda. Households in each of the selected kebeles served as second stage units. A household constitutes a person or group of persons, irrespective of whether related or not, who normally live together in the same housing unit or group of housing units and who have common cooking arrangements. A systematic random sampling was used to select the sampling units. The total sample size calculated for the district was distributed for the kebeles based on their population size; sample allocation using probability proportional to size (PPS) technique. The sampling interval was subsequently calculated using the number of households in the 10 Kebeles divided by the required number of households (i.e.6025/784=7) and the starting point (sampling unit) was determined using lottery method. Every seventh household was then eligible for inclusion. 
One individual above the age of 40 was eligible for examination from each of the selected households and a Lottery method was used when there were more than one individual in the selected households.

\section{Data collection method}

Each eligible household was visited, and a study subject selected. Sociodemographic data were filled in the questionnaire and visual acuity of both eyes separately was assessed outside the house in a shaded area. Visual acuity was measured using tumbling-E Snellen chart, and whenever the Visual Acuity was found to be worse than $6 / 9$, a pin hole was used to account for possible refractive error. Both eyes of all subjects were examined using a portable slit lamp before and after pupillary dilatation for signs of PXF in the anterior segment. PXF was diagnosed on slit lamp biomicroscopy by the presence of white dandrufflike material at the pupillary margin and/or on the anterior lens capsule of one or both eyes. Intraocular pressure (IOP) was measured using Tono-Pen (Medtronic Solan, $\mathrm{XL}$ ) before pupillary dilation. In order to estimate the width of the chamber angle, Van-Herrick method was used and when the drainage angle was judged to be not occludable (the distance between the anterior surface of the iris and the posterior surface of the cornea is more than one-fourth the corneal thickness), the pupils were dilated with $1 \%$ Tropicamide and 2.5\% Phenylephrine hydrochloride to allow examination of the lens. WHO Simplified Cataract Grading System was utilized to document about the status of the lens (17). Cataract was said to be present when nuclear, Cortical, or Posterior subcapsular standards were 2 and above. Subjects having Corneal opacification dense enough to obscure visualization of the anterior segment, evidence of active or past attack of anterior uveitis, or a history of intraocular surgery for cataract, glaucoma, or retinal detachment were excluded. Best village-based visual acuity was defined as the better of presenting and pinhole visual acuity, as measured during data collection.

\section{Statistical analysis}

Data was entered into a computer and edited for any inconsistencies before analysis using SPSS for Windows Version 15.0 to calculate means, cross tabulations and $x^{2}$ tests. Odds ratios were used to assess the odds of having increased IOP with and without PXF. Associations between PXF, demographic factors, and, other ocular diseases were computed using either the $\mathrm{x}^{2}$ or Fisher exact test, and P-values below 0.05 were considered statistically significant. Odds ratios were calculated, separately for right and left eyes, to test for associations between pseudoexfoliation and IOP, and pseudoexfoliation and senile lens changes.

\section{Results}

A total of 760 subjects were examined, giving a response rate of $96.8 \%$. Three hundred fifty-five (46.7\%) were males and 405(53.3\%) were females. The mean age was 54 (SD 9.74) (range 40-90) years. Table 1 shows the age and sex distribution of the study subjects.

Table 1. Distribution of the study subjects by age and sex, prevalence of PXF among adults in Kebena Woreda, Gurage zone; January 2017 


\begin{tabular}{|c|c|c|c|c|c|c|}
\hline $\begin{array}{c}\text { Age } \\
\text { range }\end{array}$ & $\begin{array}{c}\text { Male } \\
\\
n(\% \\
\text { column })\end{array}$ & $\begin{array}{c}\text { Men with PXF } \\
\text { (\% row) }\end{array}$ & $n$ & $\begin{array}{c}\text { Female } \\
n(\% \\
\text { column) }\end{array}$ & $\begin{array}{c}\text { Women } \\
\text { with PXF } \\
n(\% \text { row })\end{array}$ & $\begin{array}{c}\text { Total no of subjects } \\
\text { examined } \\
n(\% \text { row) }\end{array}$ \\
\hline $40-$ & 98 & $1(1.0 \%)$ & & $94(23.2 \%)$ & $1(1.1 \%)$ & $192(25.3 \%)$ \\
\hline $50-$ & 127 & 8 (6.3\%) & & $196(48.4 \%)$ & 15 (7.7\%) & 323 (42.5\%) \\
\hline $60-$ & $\begin{array}{c}(35.0 \%) \\
86\end{array}$ & $12(14.0 \%)$ & & $95(23.5 \%)$ & 23 (24.2\%) & 181 (23.8\%) \\
\hline $\begin{array}{l}70- \\
79\end{array}$ & $\begin{array}{c}38 \\
(10.7 \%)\end{array}$ & 21 (55.3\%) & & $18(4.4 \%)$ & 6 (33.3\%) & $56(7.4 \%)$ \\
\hline 80- & $6(1.7 \%)$ & $2(33.3 \%)$ & & $2(0.5 \%)$ & $2(100.0 \%)$ & $8(1.1 \%)$ \\
\hline Total & $\begin{array}{c}355 \\
(100 \%)\end{array}$ & 44 (12.4\%) & & 405 (100\%) & 47 (11.6\%) & 760 (100\%) \\
\hline
\end{tabular}

Of the total eligible population (760 subjects), 93 were found to have pseudoexfoliation in one or both eyes, a prevalence of $12.2 \%$ (95\% Cl: $9.7 \%-14.3 \%$ ). Of the 93 participants with PXF, 51(56\%) were found to have bilateral pseudoexfoliation ( $95 \% \mathrm{Cl}: 46 \%-66 \%$ ).

The mean age of participants with PXF was 63.9 years (SD 9.962, age range 40-90 years), while it was 52.63 (SD 8.90, range 40-90 years) for those without PXF. The difference was found to be statistically significant $(95 \% \mathrm{Cl}: 9.18 \%-13.11 \%, \mathrm{P}=0.00)$

Prevalence of PXF increased with age $\left(X^{2}\right.$ test; $\left.P=0.000\right)$ and was highest among subjects aged $>70$ years. Sixty-six (26.9\%) of subjects of 60 years or older had PXF in one or both eyes and $50 \%$ of subjects above the age of 70 were found to have pseudoexfoliation. Age-specific prevalence rates are shown in Table 2.

Table 2. Prevalence of PXF according to age among adults in Kebena woreda, Gurage zone: January 2017.

\begin{tabular}{|c|c|c|c|c|c|c|}
\hline \multirow[t]{3}{*}{$\begin{array}{l}\overline{\text { Age }} \\
\text { ange }\end{array}$} & \multirow[t]{3}{*}{$\begin{array}{c}\text { №․ } \\
\text { examined }\end{array}$} & $\begin{array}{l}\text { Unilateral } \\
\text { PXF }\end{array}$ & $\begin{array}{l}\text { PXF in OD } \\
\&\end{array}$ & $\begin{array}{l}\text { PXF in one or both } \\
\text { eyes, } \mathrm{n}(\% \text { row })\end{array}$ & \multirow[t]{3}{*}{$\begin{array}{c}\text { OR }(95 \% \mathrm{Cl}) \text { for PXF in one } \\
\text { or both eyes }\end{array}$} & \multirow[t]{3}{*}{$\bar{P}$} \\
\hline & & $\mathrm{n}$ (\% row) & os & & & \\
\hline & & & $\begin{array}{l}\mathrm{n}(\% \\
\text { row) }\end{array}$ & & & \\
\hline$\overline{10-50}$ & 360 & $7(1.9 \%)$ & 4 & $11(3.1 \%)$ & 1.00 & 0.000 \\
\hline $51-60$ & 283 & 19 (6.7\%) & 19 & 38 (13.4\%) & $4.7(3.7-9.7)$ & 0.000 \\
\hline ¡1-70 & 73 & $8(11.0 \%)$ & 14 & $22(30.1 \%)$ & $6.5(3.3-12.8)$ & 0.000 \\
\hline $71-90$ & 44 & $8(18.2 \%)$ & $\begin{array}{c}14 \\
(31 . \%)\end{array}$ & $22(50.0 \%)$ & $2.3(1.1-5.1)$ & 0.033 \\
\hline OTAL & 760 & $42(5.5 \%)$ & $\begin{array}{c}51 \\
51 \%) \\
(6.7 \%)\end{array}$ & $93(12.2 \%)$ & & \\
\hline
\end{tabular}


Odds ratios for the likelihood of developing either unilateral or bilateral pseudoexfoliation syndrome, by age are also displayed in the table with the 40 -50year age group as reference. The older groups all had significantly higher ORs than the reference group.

Slightly higher proportion of males (12.4\%) were found to have pseudoexfoliation in either of the eyes than females $(11.6 \%)$ which was not statistically significant $(p=0.738)$, even after Standardization for age differences ( $p=0.317$; age adjusted odds ratio, $1.29 ; 95 \%$ confidence interval, 0.79 to 2.11 ).

\section{Features of PXF}

In 68 eyes (47.2\%), deposits were visible only at the pupillary border, while in 14 (9.7\%) deposits could have seen only on the anterior lens capsule. Deposits were present both at the pupillary border and on the anterior lens capsule in 62 eyes (43\%)

\section{Intraocular Pressure}

The mean IOP of the right eye in the PXF population was 20.65(SD 5.15) (range 12-45) $\mathrm{mmHg}$ and 15.0(SD 2.30) (range 10-40) $\mathrm{mmHg}$ for the non-pseudoexfoliators. The mean difference of $5.65 \mathrm{mmHg}$ between the two populations was found to be statistically significant $(95 \% \mathrm{Cl} 4.05$ to $6.50 \mathrm{mmHg}$; $\mathrm{p}<$ 0.0001).

The mean IOP of the left eye in the PXF population was 21.69(SD 5.58) (range 12-39) $\mathrm{mmHg}$ and 15.50(SD 1.90) (range 10-24) $\mathrm{mmHg}$ for the non-pseudoexfoliators. The mean difference of $6.19 \mathrm{mmHg}$ between the two populations was statistically significant $(95 \% \mathrm{Cl} 4.88$ to $7.51 \mathrm{mmHg} ; \mathrm{p}<0.0001)$.

The mean IOP of the eye with PXF in subjects with unilateral PXF was 19.45 (SD 3.78) mmHg and, bilateral PXF eyes had a mean IOP of 21.75 (SD 5.73) mmHg. But the mean difference of $1.70 \mathrm{mmHg}$ between the two populations was not statistically significant $(95 \% \mathrm{Cl} 0.20$ to $3.67 \mathrm{mmHg} ; \mathrm{p}=0.053)$.

Fifty (34.7\%) eyes with PXF syndrome had IOP higher than $21 \mathrm{~mm} \mathrm{Hg}$. And only $12(0.8 \%)$ of eyes without PXF were found to have IOP $>21 \mathrm{mmHg}$.

Cataract was found in $59.2 \%$ of eyes with PXF, but in only $13.5 \%$ of Non-PXF eyes $(p<0.001)$, indicating a strong association between cataract and PXF. Table 3 presents data on the association between pseudoexfoliation and senile lens changes for right and left eye. In a univariate analysis nuclear and posterior subcapsular cataract were found to be significantly associated with pseudoexfoliation. $(p=0.03)$ But when adjusted for age, the association becomes statistically insignificant.

Table 3. The association between Pseudoexfoliation and senile lens changes for right and left eye among adults in Kebena Woreda, Gurage zone: January 2017 


\begin{tabular}{lcc}
\hline Status Of Lens & PXF in the right eye & PXF in the left eye \\
& $n(\%)$ & $n(\%)$ \\
\hline Clear & $15(30.8 \%)$ & $14(19.4 \%)$ \\
Nuclear cataract & $8(11.1 \%)$ & $10(13.9 \%)$ \\
Cortical cataract & $18(25.0 \%)$ & $20(27.8 \%)$ \\
Nuclear + PSC & $16(22.2 \%)$ & $12(16.7 \%)$ \\
Dense & $15(20.8 \%)$ & $16(22.2 \%)$ \\
Total & $72(100.0 \%)$ & $72(100.0 \%)$ \\
\hline
\end{tabular}

Forty-eight (33.3\%, 95\% Cl: 20.0 - 41.2) eyes with PXF had Best Village-Based distance visual acuity of $<6 / 60$. The prevalence of impaired vision in the involved eye was significantly higher in subjects with PXF (age adjusted OR, $4.55 ; 95 \% \mathrm{Cl}$ : $2.26-9.18$ ).

\section{Discussion}

This study confirms the high prevalence of PXF among the Kebena people in SNNPR, Ethiopia. The rate of $26.9 \%$ for those 60 or older in this study is even higher than highest published figures from Scandinavia, where the syndrome was first reported. The reported prevalence rate of PEX syndrome in different populations shows extensive variations- $0 \%$ in Eskimos (7), $1.6 \%$ in a south eastern US population (17), $1.8 \%$ in the Framingham Eye Study, 5-25\% in the Scandinavian countries (7), and 38\% in Navajo Indians (18).

This finding therefore suggests both genetic and environmental influences even though differences in prevalence across populations need to be interpreted with caution considering the difficulties and lack of standardization in diagnosis and the potential for sub clinical or early cases to be missed.

Two hospital-based published reports from Ethiopia described a high prevalence of PXF amongst patients with glaucoma or ocular hypertension (25.0\%) (12) and cataract (39.3\%) (13). However, this is the first population-based survey of the prevalence of PXF in Ethiopia. This study confirms the high prevalence of PXF in Ethiopia.

We found that the mean age of subjects with PEX syndrome is 11.27 years older than the normal population. Considering age specific prevalence rates, there was a significant linear increase in prevalence with age. This is also in agreement with previous reports that have shown that the prevalence of PEX increases with advancing age $(4-8,19-24)$. One remarkable finding in our results is that PXF occurs at a relatively younger age in our population (mean age being 63.9 years (SD 9.96, age range 40-90 years) compared to studies done in Iceland (mean age of 72)(24) and the Framingham study (19). This is also in agreement with findings in a hospital based study in Ethiopia (13), which has reported a mean age of $63.7 \pm 10.7$ years (range 47-91 years) in patients with PXF who were scheduled for cataract surgery. It has been suggested that persons living in lower latitudes appear to develop PEX at younger age (25).Bartholomew RS (1973): reported a prevalence rate of $6.4 \%$ in the 30-39 years age group among 
the Bantu of South Africa (26).This finding could support the theory of genetic predisposition and environmental effects in the development of PXF even though the well-known difficulty of determining age in a largely illiterate community has to be acknowledged in our study.

There are conflicting reports of gender differences in the prevalence of PXF. Women were found to be more frequently affected than men in Reikjavik eye study of Iceland and the Framingham eye studies. On the contrary, studies in Greece (20), Iran (23), and Turkey (27) have shown the reverse. We found the prevalence of PXF among men to be marginally higher than in women, but the difference was not statistically significant, which is in accordance with the findings of south Indian study (28). Probably climatic conditions provoke more or less equally intense effect in both groups of populations because females spend more of their working time in the farm land helping their husbands with farming in the district.

The finding that a reasonable number of participants with pseudoexfoliation have clinically visible deposits only over the surface of the lens emphasizes the need for dilated lens examinations preoperatively.

Like many of population based studies $(20,21,22,29)$, we found IOP levels to be generally higher in eyes with pseudoexfoliation than in eyes without it. The mean IOP in subjects with PXF was $5.27 \mathrm{~mm} \mathrm{Hg}$ higher than in those without PXF which was significant.

High IOP (IOP>21 mmHg) was recorded in $36.1 \%$ of subjects with PXF compared to $0.8 \%$ in subjects without PXF. The PXF population had a significantly increased prevalence of IOP over $21 \mathrm{mmHg}$. Although investigation for evidences of glaucoma is beyond the scope of this study, it seems that a significant number of subjects with PXF were found to have one of the major identified risk factor for glaucoma, i.e. ocular hypertension.

The increasing prevalence of PXF and cataract with age and the association of PXF with the most common type of cataract (nuclear and PSC cataract) have public health implications for our country. Improved healthcare results in a definite demographic shift toward aging that may result in a higher burden of both PXF and cataract. Eyes with PXF have a greater frequency of complications such as zonular dialysis, capsular rupture, and vitreous loss at the time of cataract extraction. The surgical procedure is more difficult because the pupil may not dilate well. It has also been shown that PXF patients have an increased risk of acute increase in IOP after cataract surgery. Postoperative complications of posterior capsular opacification; capsule contraction syndrome, intraocular lens decentration, and inflammation are also greater in eyes with PXF.

A preoperative diagnosis of PXF and appropriate precautions during surgery may help to reduce the frequency of complications.

Recent studies from India (21) and Burma (22) have highlighted the association between PXF and visual morbidity rates. Similarly, in our study blindness was strongly associated with the presence of PXF in the 
univariate analysis. We found that $30.6 \%$ of those with PXF were blind, marginally higher than data reported in the Aravind Comprehensive Eye Study (ACES) from a different part of southern India (25.7\%) and The Andhra Pradesh Eye Disease Study (20.5\%).

\section{Conclusion And Recommendations}

Our study confirms that there is a high prevalence of PXF in our population associated with a relatively higher average IOP. With those findings the following measures are recommended: IOP should routinely be measured objectively in every case of PXF as there is a high chance that ocular hypertension and glaucoma be associated with. A guide line should also be developed about the special precautions that should be undertaken by cataract surgeons while managing patients with cataract with PXF. A high degree of suspicion should be maintained for the presence of PXF during routine ophthalmic examination, particularly in the elderly with high IOPs.

Further studies are recommended to scrutinize the reason why relatively younger adult are affected more frequently in our population and identify the cause of poor vision in patients with PXF, review the relationship between PXF and glaucoma in our population, and identify the conversion rate of unilateral PXF to bilateral PXF and development of ocular hypertension over a certain period of time.

\section{Declarations}

\section{Ethical approval and consent to participate}

The study was conducted following the Kelsinki declaration and after it was approved by the research ethical committee of School of Medicine, Addis Ababa University. Informed verbal consent was taken and only those who consented were studied.

\section{Consent for Publication}

The manuscript represents original and valid work and that neither this manuscript nor one with substantially similar content under our authorship has been published or is being considered for publication elsewhere, except as described in the journal's submission form and cover letter submitted with the manuscript, and copies of closely related manuscripts have been provided. We agree to be accountable for all aspects of the work in ensuring that questions related to the accuracy or integrity of any part of the work are appropriately investigated and resolved; and If requested. All identifying information of subjects involved in the study has been appropriately anonymized.

\section{Availability of data and material}

We will provide the data or will cooperate fully in obtaining and providing the data on which the manuscript is based for examination by the editors or their assignees; and I, as the corresponding author, agree to serve as the primary correspondent with the editorial office, to review the edited manuscript. 


\section{Competing interests}

- The authors have nothing to disclose.

\section{Funding}

- Orbis International financially supported the field data collection.

\section{Authors' contributions}

The three authors have made substantial contributions to the intellectual content of the paper by:

- concept and design acquisition,

- analysis and interpretation of data.

- drafting the manuscript critical revision and

- statistical analysis, and direct supervision of the data collection process. Acknowledgements

\section{Acknowledgement}

This study was conducted with the financial support of ORBIS International Ethiopia. We are indebted to the kindness of all the staffs who were unduly supportive in all ways throughout the study period.

We are also grateful to Drs. Abebe Bejiga, Dawit Wondimagegn and Solomon Shiferaw for their invaluable comments on the design of the study and for reviewing the manuscript.

\section{References}

1. Netland PA, Ye H, Streeten BW, Hernandez MR. Elastosis of the lamina cribrosa in pseudoexfoliation syndrome with glaucoma. Ophthalmology 1995; 102:876-886.

2. Ritch R. Exfoliation syndrome: the most common identifiable cause of open angle glaucoma. $J$ Glaucoma 1994; 3:176- 178.

3. Taylor HR, Hollows FC, Moran D. Pseudoexfoliation of the lens in Australian aborigines. Br J Ophthalmology 1977; 61:473- 475.

4. Cashwell LF Jr, Shields MB. Exfoliation syndrome in the southeastern United States, I: Acta Ophthalmol Suppl 1988; 184:99-102.

5. Sveinsson K. The frequency of senile exfoliation in Iceland: fibrillopathy or pseudoexfoliation. Acta Ophthalmol 1974; 52:596-602.

6. Sood NN. Prevalence of pseudoexfoliation of the lens capsule in India. Acta Ophthalmol 1968; 46:211-214.

7. Forsius H. Prevalence of pseudoexfoliation of the lens in Finns, Lapps, Icelanders, Eskimos, and Russians. Trans Ophthalmol Soc U K 1979; 99:296-298. 
8. McCarty CA, Taylor HR. Pseudoexfoliation syndrome in Australian Adults. Am J Ophthalmol 2000; 129:629-633.

9. A L Young, W W T Tang, and D S C Lam. The prevalence of pseudoexfoliation syndrome in Chinese people. Br J Ophthalmol 2004; 88(2): 193-195.

10. Guzek JP, Holm M, Cotter JB. Risk factors for intra operative complications in 1000 extracapsular cataract cases. Ophthalmology 1987; 94:461-466.

11. Lowe RF. Primary angle-closure with capsular exfoliation of the lens. Br J Ophthalmol 1964; 48:492494.

12. Bedri A, Alemu B: Pseudoexfoliation syndrome in Ethiopian glaucoma patients. East Afr Med J 1999, 76:278-280.

13. Teshome T, Regassa K. Prevalence of pseudoexfoliation syndrome in Ethiopian patients scheduled for cataract surgery; Acta Ophthalmol Scand. 2004: 82: 254-258.

14. Bora S, Alemu B. Clinical Profile of chronic glaucoma at initial presentation to Menelik Hospital. Unpublished

15. Alan P. Rotchford, James F. Kirwan, Gordon J. Johnson, Paul Roux, Exfoliation Syndrome in Black South Africans: Arch Ophthalmol. 2003;121:863-870.

16. Who cataract grading group, Thylefors et al. Ophthalmic Epidemiology, 9(2): 83-95 (2202)

17. Cashwell LF, Shields MB. Exfoliation syndrome: prevalence in a southeastern United States population. Arch Ophthalmol 1988; 106:335-36.

18. Faulkener HW. Pseudoexfoliation of the lens among Navajo Indians. Am J Ophthalmol 1971; 72:206

19. Rita Hiller, Robert D. Sperduto, E. Krueger. Pseudoexfoliation, Intraocular Pressure, and Senile Lens Changes in a Population-Based Survey: Arch Ophthalmol 1982; 100:1080-1082)

20. Vassilios P. et. al. Epidemiology of pseudoexfoliation in the island of Crete (Greece): Acta Ophthalmol. Scand. 1997: 75: 726-729

21. Thomas, Praveen Kumar Nirmalan, and S. Krishnaiah; Pseudoexfoliation in Southern India: The Andhra Pradesh Eye Disease Study; Investigative Ophthalmology \& Visual Science, April 2005, Vol. 46, No. 4, 1171-1176.

22. A M Abdul-Rahman. et. al.; Pseudoexfoliation in a rural Burmese population: the Meiktila Eye Study; Br J Ophthalmol 2008; 92:1325-1328.

23. Nouri-Mahdavi, N. Nosrat, R. Sahebghalam and M.Jahanmard, Pseudoexfoliation syndrome in central Iran: A population-based survey. Acta Ophthalmol. Scand. 1999: 77: 581-584

24. Arsaell Arnarsson et. al. Pseudoexfoliation in the Reykjavik Eye Study: prevalence and related ophthalmological variables; Acta Ophthalmol. Scand. 2007: 85: 822-827

25. Ringvold A., Epidemiology of the pseudoexfoliation syndrome. Acta Ophthalmol Scand. 1999: 77: 371-375.

26. Bartholomew RS (1973): Pseudocapsular exfoliation in the Bantu of South Africa. Br J Ophthalmol 57: 41-45. 
27. Yalaz M. et. Al; The frequency of pseudoexfoliation syndrome in the Eastern Mediterranean area of Turkey; Acta Ophthalmologica: 70 (1992) 209-213

28. Arvind H, Raju P, Paul PG, et al. Pseudoexfoliation in South India. Br J Ophthalmol. 2003; 87:1321-3.

29. Alan P. Rotchford, James F. Kirwan, Gordon J. Johnson, Paul Roux, Exfoliation Syndrome in Black South Africans: Arch Ophthalmol. 2003; 121:863-870.

\section{Figures}

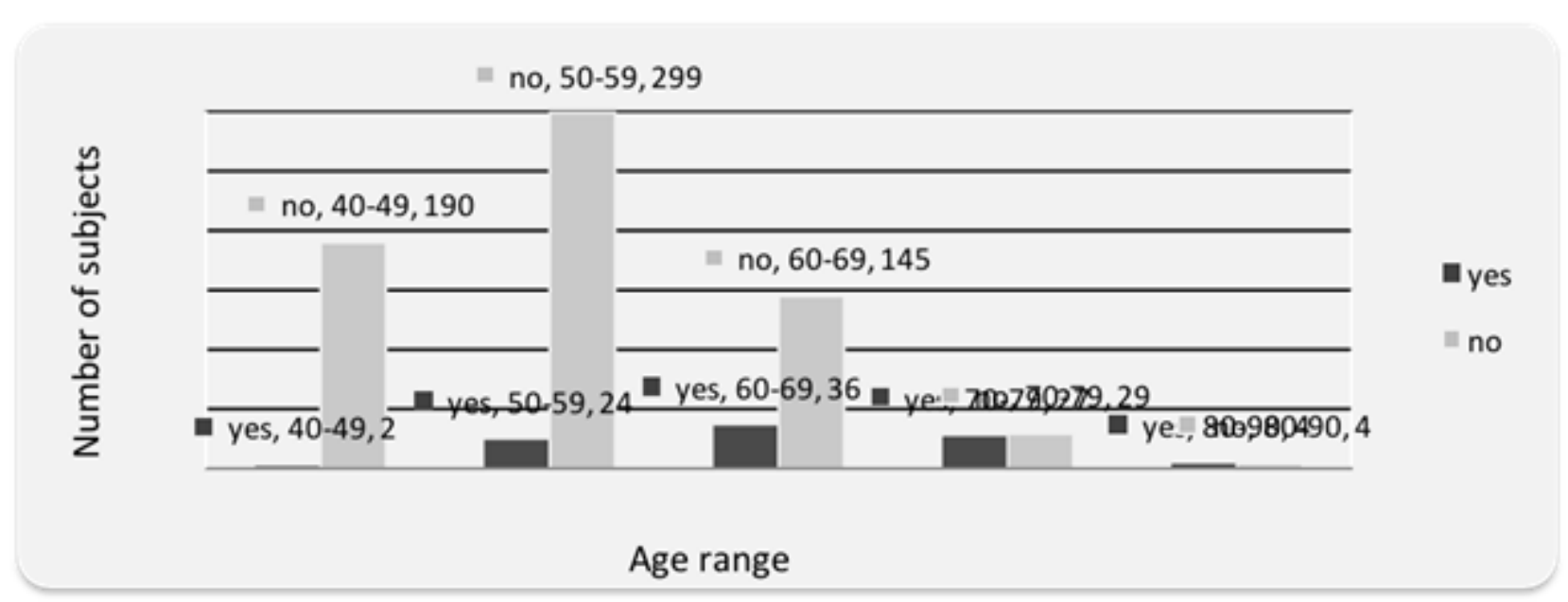

\section{Figure 1}

Distribution of PXF by age among adults in Kebena Woreda, Gurage zone, January 2017 